Ministry of Health Recep Tayyip Erdogan University Training and Research Hospital, General Surgery Department, Rize, Turkey

Corresponding Author:

Gokhan Demiral, MD

General Secretary, Rize Public Hospital Association

Rize Public Hospital, 1st floor

53100 Rize, Turkey

E-mail: drgokhandemiral@yahoo.com

Received: 06.10.2017,

Accepted: 27.12.2017

DOI: $10.5799 /$ jcei.413076

\title{
An incarcerated Amyand's Hernia: Shall we Apply Appendectomy Routinely?
}

\author{
Gökhan Demiral, Muhammed Kadri Çolakoğu, Süleyman Kalcan, Ahmet Pergel,
}

\section{A B S T R A C T}

Amyand's hernia is a very rare clinical condition characterized by the presence of a normal or inflammed appendix within the inguinal hernia sac. It may be present as an acute apendicitis inside the sac or incarcerated hernia. Sometimes it may be asymptomatic. We report a case of 70-year-old male patient that was presented to our emergency service with a huge right inguinal mass that was diagnosed as an incarcerated inguinal hernia and underwent operation. The intraoperative findings included small intestinal segment, large omental tissue and mobile cecum with healthy appendix inside the sac. There was no sign of strangulation. Lichtenstein herniorhaphy was done without appendectomy.

Key Words: Amyand's hernia, incarceration, appendix vermiformis

\section{INTRODUCTION}

An appendix vermiformis in an inguinal hernia sac, with or without appendicitis, is called Amyand's hernia [1]. Claudius Amyand in the year 1735 performed appendiceal resection in treatment of this case [2]. In his honor, inguinal hernia containing appendix vermiformis has been named after him. It is an extremely rare condition, non-inflamed appendix in the hernia sac can be seen up to $1 \%$ of all inguinal hernias [3]. In this study we aim to present a 70-yearold incarcerated Amyand's hernia patient.

\section{Case Report}

A 70-year-old-male patient was presented to our clinic with a huge right inguinal mass which had been protruding for 5 years prior to hospital admission. He stated that the mass increased in size when standing and before defecation but decreased in size after stool passage or lying down. Severe tenderness had been noted for 3 days and the mass was not reducible recently. Additionally, he had experienced urinary frequency and nocturia.
In his medical history, hypertension with regular drug control was found out. The patient was interned for surgical intervention with the diagnosis of incarcerated inguinal hernia.

Laboratory tests were within normal limits. Blood pressure was controlled. Patient was taken to the operating room for emergent surgery and underwent a general anesthesia. A right oblique inguinal incision was used. The surrounding adhesions were rigorously dissected and herni sac was opened. Small intestine, large omental tissue, mobilized cecum with ascending colon and a normal appendix (Figure 1) were explored within the indirect incarcerated herni sac arising from a narrow internal inguinal ring. There was no sign of strangulation. All the component reducted to abdominal cavity. Herniorhaphy was done by Lichtenstein method with polypropylene mesh. Appendectomy wasn't done. Postoperatively patient's condition was uneventful and he was discharged on the next day with normal intestinal activity. He was followed up at our clinic without any further complication. 


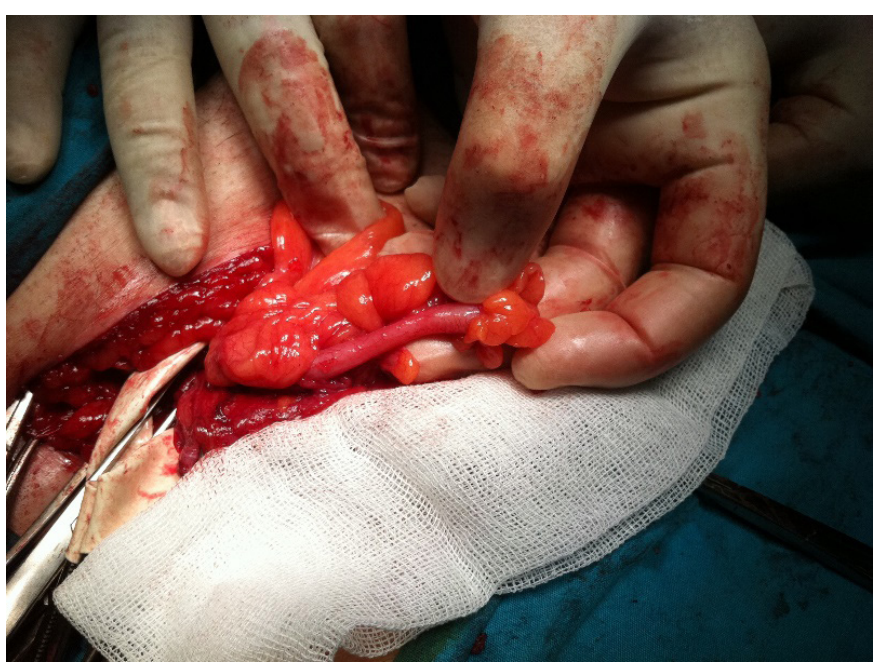

Figure 1. The appendix vermiformis that was found in the right inguinal hernia sac.

\section{DISCUSSION}

Amyand's hernia is defined as non-inflamed appendix in an inguinal hernia [4]. There are different clinical presentations of Amyand's hernia. Clinically patient may be presented with reducible or incarcerated hernia with a non-inflamed or inflamed appendix. Ingested foreign body (metallic or non-metallic) may be in the appendix causing perforation or not [4]. Presence of appendicitis in the hernia sac is much less common and is seen in $0.08 \%$ of all cases [3,5]. Most of the cases of Amyand's hernia have occurred in the right inguinal region; only a few cases have been reported in the left [6]. Our patient was presented with right huge inguinal mass as incarcerated hernia.

In the treatment of Amyand's hernia some procedures are being discussed including traditional hernioplasty, mesh hernioplasty and endoscopic hernioplasty [6]. The necessity for appendectomy is questionable when a noninflamed appendix is being found [7]. In the case of a normal appendix, incidentally found within the hernia sac, prophylactic appendectomy procedure with the hernia repair is not recommended by many surgeons. Reduction of the appendix and mesh hernioplasty are favoured [8,9]. Because of the high risk of infection of prostetic mesh material, authors agree that if there is an inflamed appendix, the repair of the hernia should be performed with Bassini or Shouldice techniques, without making use of synthetic meshes or plugs within the defect [10-12]. Wound infection around the operation site may increase the morbidity and may also cause hernia recurrence [1]. Losanoff and Basson have introduced four basic types of Amyand's hernia claiming that each should be treated differently $[11,13]$. This is the most widely accepted classification that summarizes operative findings and management. In type 1 , a normal appendix is found within an inguinal hernia. Hernia reduction with mesh repair and appendectomy in young patients are the treatment of choice. Type 2 includes an inflammed appendix within an inguinal hernia, without signs of peritonitis and abdominal sepsis. Appendectomy through herniotomy with primary repair of hernia with Bassini or Shouldice technique (no mesh) is proposed. In type 3, peritonitis with abdominal sepsis exists. Laparotomy, appendectomy and peritoneal lavage and subsequent hernia repair without mesh are indicated. Finally, in type 4 , some other related or unrelated abdominal pathology exists. A laparotomy in terms of identification and treatment of this pathology should be performed. Appendectomy and hernia repair without mesh should follow [11]. Most surgeons agree that the presence of acute appendicitis (Losanoff-Basson type 2,3,4) within a hernia should be a contraindication for the use of synthetic meshes or plugs. However, there is a conflict among surgeons whether or not to perform an appendectomy in type $1[8,9]$. In our case, a type 1 Amyand's hernia, there was no any sign of appendicitis and we applied mesh hernioplasty without appendectomy.

Preoperative diagnosis in this unusual condition is very difficult. Computed tomography may be useful in diagnosis but are not performed routinely. Although our patient was presented as an emergency case and strangulation was considered during the preoperative period, we did not order abdominal CT scanning [6]. As in our case almost all surgeons make the diagnosis intraoperatively. Strangulated hernia, strangulated omentocele, Richter's hernia, testicular tumor with hemorrhage, acute hydrocele, inguinal adenitis, and epididymitis should be thought on differential diagnosis $[6,14]$.

In conclusion, Amyand's hernia is a very rare unexpected situation that may be diagnosed incidentally during an inguinal hernia operation and every surgeon should consider the possibility of coping with Amyand's hernia. The decision as to whether surgeon should perform appendectomy or only hernioplasty is case relevant. As a result, we suggest that the decision to apply appendectomy and/or use prosthetic mesh material should be decided according to the intraoperative surgical findings.

Conflicts of Interest: None declared.

Financial Disclosure: No financial support was received.

\section{REFERENCES}

1. Psarras K, Lalountas M, Baltatzis M, et al. Amyand's hernia- vermiform appendix presenting in an inguinal hernia: a case series. J Med Case Rep. 2011;5:463.

2. Amyand C: Of an inguinal rupture, with a pin in the appendix coeci, incrusted with stone; and some observations on wounds in the guts. Phil Trans Royal Soc. 1736;39:329.

3. Thomas WEG, Vowles KDJ, Williamson RCN. Appendicitis in external herniae. Ann R Coll Surg Engl. 1982;64:121-2.

4. Llullaku SS, Hyseni NS, Kelmendi BZ, et al. A pin in appendix within Amyand's hernia in a six years old boy: case report and review of literature. World J Emerg Surg. 2010;5:14.

5. D'Alia C, Lo Schiavo MG, Tonante A, et al. Amyand's hernia: case report and review of the literature. Hernia. 2003;7:89-91.

6. Kwoka CM, Suc CH, Kwangb WK, Chiu YCal. Amyand's hernia-Case Report and Review of the Literature. Case Rep Gastroenterol. 2007;1:6570. 
7. Hutchinson R. Amyand's hernia. J R Soc Med 1993;86:104-5.

8. Milanchi S, Allins AD: Amyand's hernia: history, imaging, and management. Hernia. 2008;12:321-2.

9. Sharma H, Gupta A, Shekhawat NS, et al. Amyand's hernia: a report of 18 consecutive patients over a 15-year period. Hernia. 2007;11:31-5.

10. Ballas $\mathrm{K}$, Kontoulis $\mathrm{T}$, Skouras $\mathrm{C}$, et al. Unusual findings in inguinal hernia surgery: Report of 6 rare cases. Hippokratia. 2009;13:169-71.

11. Losanoff JE, Basson MD. Amyand hernia: a classification to improve management. Hernia. 2008;12:325-6.
12. Livaditi E, Mavridis G, Christopoulos GG. Amyand's hernia in premature neonates: report of two cases. Hernia. 2007;11:547-9.

13. Losanoff JE, Basson MD. Amyand hernia: what lies beneath a proposed classification scheme to determine management. Am Surg. 2007;73:128890.

14. Logan MT, Nottingham JM. Amyand's hernia: A case report of an incarcerated and perforated appendix within an inguinal hernia and review of the literature. Am Surg. 2001;67:628-9. 